\title{
Understanding Muslims Self-Presentation Strategies in Online Dating
}

\author{
Adamu Abubakar', Nur Irdina Mohd Noorani², Ummu Syafiqah Mohd Rashidi ${ }^{3}$ \\ 'Dept. of Computer Science, KICT, International Islamic University Malaysia. \\ ${ }^{2,3}$ Dept. of English, KLM, International Islamic University Malaysia. \\ adamu@iium.edu.my
}

\begin{abstract}
This paper investigates the factors influencing online self-presentation strategies on dating platforms, and explore how Muslims users manage their online presentation on dating approach -associated with Muslim-oriented dating application and mainstream-oriented dating application in order to accomplish the goal of finding partner. Twenty-profiles from Tinder and MusMatch active online dating user was used for the study. Qualitative content analysis was used to explore the approaches of interactions associated with individual self-presentation. Four main themes were generated: Screen names, Terminal identities, Net Presence and Personal Profile. The findings reveal that both MusMatch and Tinder allow its users to choose their own screen names without any restrictions. The platforms showed how screen names can be used as a strategy to present certain impression. Users tend to post their personal interests in order to attract their potential partners on Tinder as well as MuzMatch. Users of MuzMatch used acronyms to identify themselves, whereas, users of Tinders are free to construct a socially desirable identity that may attract people's attention and publicity. The frequency with which MusMatch users appear to interact for relationship that leads to marriage is far more than any other form of relationship. Tinder users are free to post anything as their profile picture, whereas in MuzMatch it requires authentic profile pictures. MusMatch provision for establishing relationship requires that a chaperone should be allow to mediate interactions among the potential partners in order to preserve Muslims-oriented dating style.
\end{abstract}

Keywords— Online Dating, Self-presentation, Net presence, Terminal Identity.

\section{INTRODUCTION}

This era of Information technology placed more value into an individual's online identities as opposed to their offline or real-life identity. Everyone can be known online based on how he/she present himself/herself. In this fast-paced world, the construction of identity is being influenced by the existence of the new media as people are more engaging in those platforms in order to communicate with each other [1]. There is an emerging pattern where people no longer accept their offline lives as their real identity but associate themselves more with their online personas [2]. Social media is one of the platforms for socializing. It is a collection of "apps and websites-that provide users with digital environments in which they can send and receive digital content or information over some type of online social network" [1]. Some of these platforms are specific for dating, where they represent an opportunity to mediated dating or relationship formation [3]. Eventually, lead to a two steps relationships, where people meet online first, and then later agree to move offline.

Online dating allows users to "showcase" their living experiences which make them play certain characters in the social arena [4]. Social media content are mostly photographs and videos that users post to present their online self. Similarly, online dating platforms uses these data formats as their main content. Online Self-presentation is generally motivated by a desire and interest in making a favourable impression for others, or given an impression that corresponds to the individual's ideals [5]. Here, the concept of identity is changing as it is affected by the emergence of these social platforms. However, with the existence of the new media, identity construction has moved into a virtual sphere. As a result, a person may be tempted to construct a false self-presentation in order to be liked more by their peers or according to their own preferences. That is why the Internet was conceptualized as a social laboratory, where self-presentation might be built on the false constructs to consists of the concepts of what is appealing, good and based on one's interest [6]. That means, all of the aspects of one's true self that is not conform to these notions may be put aside and rejected by the individual.

This study focus on two main categories of Online Dating platform namely: Muslim-oriented and mainstream-oriented online dating platforms. For the mainstream online dating platform include "Tinder", "Tingle" and "Blendr" whereas for the Islamic-based online dating include "MuzMatch", "Muslima" and "Malaysian Cupid". However, this study dwells on analysing Tinder as the mainstream-oriented online dating platform and MuzMatch as the Islamic- 
oriented online dating platform in order to conduct a focused and in-depth study within the Malaysian context.

Tinder was launched in 2012, as one of the most popular online dating platform with over 2.6 billion "swipes" per day and producing about 26 million matches [7]. It was made available for use to about 190 countries worldwide. Tinder and the likes are generally associated with casual sex. This is because many people join the platform with the intent of seeking for casual sex partners and few look for serious relationships [8]. It is a new way for emerging adults to initiate committed romantic relationships. Tinder works by allowing users to set up their profiles and specify who or what they are interested in looking for. Users can share their information such as their pictures, occupation, past educations and they can also connect to their Instagram and Spotify accounts to disclose more information. It also detects their location and connects them to users that are nearby their area [9]. Finally, it allows for establishing relationships by "swipe right" to each other's account. The application provides a feature whereby users can swipe right on another user's account which indicates that they are interested to know the person while swiping left indicates otherwise. Prior to swiping the other user's account, they may view the user's profile information. Apart from having private conversations between two users, Tinder also allows users to join groups and hence communicate with more than a user at one time. The group works similar as how a private chat works which is by swiping right the group that they are interested to join [7].

MuzMatch on the other hand is meant for Muslims and available for used for about 190 countries, with about 3000,000 members. It was launched in 2015 and it claims to cater to Muslims who are seeking for serious relationships that will leads to marriage. This is due to the difficulty of Muslims in seeking life partners for marriage using the traditional methods [10]. As of 2020, MusMatch mediated over 60,000 Muslim weddings worldwide. MuzMatch has some similar features with Tinder and also many differences which are interesting to highlight on. The "swipe" function to get a match is similar to Tinder and users can customize their profile information. Not only that, MuzMatch also identifies the users' location and provides matches that are nearby. However, MusMatch allows users to blur their pictures and use a nickname. The users get to make their own decision if they want to reveal their picture and real name. It reminds the users to engage according to Islamic rules when they are using the platform. MusMatch has a "chaperone" feature which allows users to include another person such as their family members or friends to supervise the conversation. This is in line with the Islamic guideline whereby a man and a woman should not be left alone in proximity without a guardian. Users can give positive feedbacks to the person they chatted with. The positive feedbacks will result in profile badges as a reward for good behaviour. As a safety measure, MusMach provide Selfie Verification in which the profiles are all verified manually using Selfie Verification, SMS confirmation and GPS location.

These two online dating environments described above allows people to present different types of roles depending on whom they are engaging with, through a particular post, status, comment, photo or a video. It is clearly shown that online self-presentation may change according to their setting and platform where one needs to face the need to reclaim or redefine themselves in the new environment. Considering this, the current study explores how individuals present themselves on the online dating platforms, and analyse the factors affecting the use of mainstreamoriented and Muslim-oriented online dating platforms.

\section{RELATED WORK}

Self-representation is known as the visible signs created on the screen in order to represent and manifest the users of the platform. It is clear that Internet users shape their self by presenting the most valued aspect of themselves in their social interactions as to be perceived according to their intention. Previous research study reveals contrasting views on self-presentation of individuals in the cyberspace [2-6]. Crucial to point out that individuals mostly describe their body features online, what they like or dislikes, their interests, and accomplishments [11]. Similarly, Jacobson, et al. [12] reveals that men and women choose to represent themselves on online dating sites using everything that will pleased them. The analysis of how people disclose different amount of information on their online dating profiles, reveals that in general, users present very brief statements about themselves on Tinder which only discloses a small glimpse of their personality. Men mostly expressed themselves regarding work, sports and the military while in contrast, women shared information about work, school and social interests. It was also revealed that women's descriptions and photos were not very clear while men were more direct. Furthermore, it was indicated in Sumter et al. [8] love motivation of users appeared to be a stronger motivation to use Tinder than the Casual Sex motivation, but men were more likely to report a Casual Sex motivation for using Tinder than women.

Turner [13] conducted a study on self-presentation of women's dating profiles and explored how women used their profiles on online dating sites as a form of selfpresentation. It was revealed that the online features in terms of content and structure of the women's selfpresentation in searching a romantic partner online are in two categories namely: "Single women" and "those in relationships". That is, it was discovered that women in relationships stills uses online dating platform. However, the Single woman profile, are characterized by Poetic and 
Philosophical, Idealistic, Independent and Active presentation, while those women in relationships, present their "Relationship Story" mostly displays adventures of a "New Life Stage" and "Sharing a Life with Someone".

Ellison et al. [14] reveals that women who emphasized on their potential partner (Who You Are), are having more control of their self-presentation in online contexts. In this case, the women showcased being more particular in choosing their potential partner as well as having more control of their love life.

Similarly, Morganet al. [15] also discovered that both women who are single and those in relationships are interested in dating those that have similar interests or attributes of always asking for "who are you" first. That women utilizes self-presentation on the descriptions of their profile by focusing on themselves and a potential partner.

Ranzini and Lutz [16] studied how Tinder users present themselves and their personality characteristics through a quantitative online survey of sample of 497 respondents from the United States. It was found that self-esteem is the most important factor which increases real selfpresentation and decreases deceptive self-presentation. Even though, Tinder is perceived to be a platform with majority of heterosexual users, homosexuals are pressured to limit their visibility in the application [17]. However, presenting a deceptive version of oneself could help those with low self-esteem [18]. Thus several other motives for using Tinder were analysed to be influenced by relationship status, sex, friendship, travelling, self-validation and as a source of entertainment. Tinder users may portray themselves in their true self or deceptive self which is influenced by several factors but the most dominant factor is self-esteem.

Ward [19], reveals how people control the way they present and disclose themselves to their potential partners. Self-disclosure is the act of revealing and telling people about one's personal information to another. In the virtual environment self-presentation and self-disclosure are important. The finding of the study indicates that users who are serious in seeking intimate relationship, will be more truthful about their appearance as well as increasing their self-disclosure towards their online potential partner.

Majority of the previous studies in the area of the current study presents self-presentation online, and some where very specific to Tinder. Unfortunately, self-presentation among Muslims-oriented online dating platform as compare to the mainstream platforms has not been greatly discussed. Self-presentation of users in mainstream-oriented online dating platform might be different from those in Muslimsoriented platform. In both cases, there might be deceptive presentation due to the online setting. Therefore, this paper presents a study on Tinder's users and MusMatch users in order to understand how they present themselves and disclose information's.

\section{METHODOLOGY}

This study explores the approaches of interactions associated with individual self-presentation for the mainstream-oriented and Muslims-oriented online dating platforms. In order to achieve the objective of the study, qualitative content analysis techniques. This form of study involves analysis of the content or information and symbols in written documents or other communication media, including websites is adopted [20]. The research population of this study consisted of Muslim users of the two dating platforms (Tinder and MuzMatch). According to White and Marsh [21], the sampling of a qualitative content analysis should be theoretical and purposive in order to achieve the objective of the study. As a result, for quota sampling, an equal number of profiles from both platforms were selected: 10 were from Tinder and another 10 from MuzMatch with five males and five females each. According to Showkat and Parveen [22], quota sampling is ideal to investigate subgroups and in this study, the subgroups of interest were users of Tinder and MuzMatch. The personal information of the respondents was kept confidential. The total of the 20 profiles of users of the two online dating platforms were selected for analysing purposes. The data were collected through both Tinder and Muzmatch. This study focused on the elements used to present the users such as their screen names, terminal identities, net presence and personal profiles.

First, 10 profiles from Tinder were carefully selected based on their religion and description on their profiles. This is because the study seeks to analyse how Muslims present themselves on the dating sites, hence only profiles of Muslims were chosen as the participants of this study. As for the descriptions on their profiles, only those with minimum of 20 characters were analysed in order to provide sufficient information for data analysis. This is related to homogeneous purposive sampling as it focuses on a particular subgroup in which all the samples have certain similar characteristics [23-24].

Second, the same process was repeated in the MuzMatch application. Third, the content of the profiles was analysed and several themes were identified. Fourth, the themes were broken down into codes. Codes are important to record content from text into quantitative data. Fifth, the codes under the themes were compared between the two applications to identify similarities and differences. Finally, after thorough analysis, four main themes were discussed which are their terminal identities, net presence, screen names and personal profiles. This is to ensure that the data collected provided sufficient information for data analysis as the objective of this study is to analyse self-presentation of 
Muslims in particular, on different types of online dating platforms.

\section{ANALYSIS AND PRESENTATION OF THE RESULTS}

This paper dwells on "How Muslims present themselves in a Muslim-oriented online dating platform and a mainstreamoriented online dating platform". The approach of the study relies on two dating platform (MustMatch and Tinder). They were analysed and the analysis yielded four themes which are "screen names", "personal profiles", "net presence" and "terminal identities" as the factors influencing the use of both dating platforms. The impact of each themes are discussed in detail in the proceedings subsections.

\section{A. Screen Names}

Both Tinder and MuzMatch allow its users to choose a username without any restrictions. It was found that Muslim users on both sites displayed similarities and a difference in how they portray their screen names on the two platforms. First name, full name, and nicknames are used as screen names on both platforms while acronyms are also used as screen names on the MuzMatch application. The differences in the use of screen names can be related to how screen names can be used as a strategy to present a certain image of oneself according to their personal motives [25]. This result is similar to the study performed in Whitty [26], indicating that choosing the right name is important especially on a dating site as it may attract others to the profile.

First names such as Fikry, Syed and Aisyah are used on both dating applications by men and women. Not only that, some users also used their full name which consists of a first, middle and last name such as Mohd Izzudin Helmi. This could be due to concealing an individual's identity on online dating sites being counterproductive towards the purpose of such dating sites, which is to allow individuals to get to know each other. This is supported by the study of Cobb and Kohno [27] who found that participants of online dating sites agreed that several information such as name and location of the person they are going to get to know are important for the other party. Apart from real names, nicknames are also commonly used among users from both dating sites. Nicknames were used by users of both Tinder and MuzMatch by taking an essence of their real names and shortening it into a single pronunciation such as Niq, Fie and Faz. Another type of nickname observed on the dating applications involve the combination of a person's name and a trait or characteristic; najwakiut. This type of nickname is classified under Personal Character on Bechar-Israeli's [28] types of Nicknames and is rated as low anonymity as well. The use of this nickname can be related to the term 'foggy mirror' whereby users create a nickname which they thought to reflect their own personality which is valid for online dating chats [14]. An example of this is the username hot Latina which the author explained as having the need to highlight one's positive attributes to attract other participants' attention.

However, a distinct dissimilarity in the usage of screen names between the two platforms were identified, whereby only users on MuzMatch used acronyms to identify themselves. According to Lakaw [29] this type of nickname is the highest level of anonymity online and it is categorised as Unknown in Bechar-Israeli's [28] types of Nicknames. Other than to express anonymity, the author explained that it contains a certain message or purpose such as the initials of the individual's real name.

\section{B. Personal Profiles}

Personal profiles are the description box that allow users of both Tinder and MuzMatch to express themselves as a form of introduction of their personal self that will be read by other users before deciding to converse with them. From the profiles analysed, it was found that there are a number of similarities and also significant differences on how the users of these two platforms use this feature to present themselves.

A common trait is that some men and women on both dating platforms wrote very short descriptions of themselves. Some included an information about themselves such as their interests and personal attributes while some only wrote puns or a simple 'nothing much'. This can be related to the Social Penetration Theory, whereby the process of getting to know a person is described as peeling an onion. The surface, general information is revealed first, and the deeper layers are revealed later when a mutual connection has been made between both parties.

Tinder and MuzMatch operates in a way which partners can only engage in a conversation when both parties are mutually interested (by swiping right for Tinder and pressing the mark sign for MuzMatch). In contrast, there are also a number of users who wrote very long and detailed descriptions of themselves, their interests and their intention, especially on MuzMatch. This is supported by Jacobson et al. [12] that users tend to express a willingness to expose themselves in an overwhelming manner online compared to face-to-face situations due to the absence of physical presence. Similarly, Whitty [26] discover that people reveal more depth and breadth of information about themselves in their online dating profiles compared to in face-to-face dating atmosphere. This is important because starting relationships involves significant decisions related to the appropriate information which should be disclosed as well as the method of disclosure which would create a favourable impression. This eventually leads to the process of 'peeling the onion' or self-disclosing much sooner compared to in real life situations. In addition, Ellison et. al, [14] reported that more self-disclosure about a person increases the credibility of their self-presentation, while 
Whitty [26] agreed to increase in the probability of finding a more compatible partner as they can easily find those with common interests or traits which leads to the next point that will be discussed.

Next, personal interests such as travelling, photography, hobbies and personal traits like funny and creative are also mostly shared among users of the two dating platforms. This is because people tend to look for relationship partners that have the same interests or characteristics as them [30]. Apart from disclosing information about oneself, some of the users of both platforms also shared what they are looking for in a partner or relationship. For example, "looking for a fun, easy going and loyal partner".

In both Tinder and MusMatch, some of the users shared their physical attributes such as height, facial features and skin colour. This is because physical characteristics are important in influencing the judgement of other users. That is, self-rated physical attractiveness is one of the most important qualities for men and women in selecting a partner on online dating sites [31]. This is further described by Finkel et al. [32], that experiential attributes such as rapport and sense of humour are best evaluated face-toface, which leaves searchable attributes that can be identified through simple information on online dating profiles such as physical attributes. Hence, these searchable attributes should be fully utilised by the users of online dating platforms in presenting themselves to other users.

Besides that, the users of both dating platforms also shared their Instagram username, WeChat username and even their personal mobile number so that others can find them on other platforms. This could be to increase their credibility as supported by Ward [19] who conducted a study on self-disclosure in Tinder and found that users often attempt to locate the Facebook profile of their Tinder match to validate the authenticity of the user. It functions as a check to the match's self-presentation and perceived attractiveness as the pictures on Facebook might vary from their Tinder account. Significant differences can also be identified on self-presentation of users of Tinder and MuzMatch.

First, there are certain added features on MuzMatch that users are required to answer which are not present on Tinder which is related to religion. The questions are regarding their Islamic Sect (Sunni and Shi'ite), Status (Single, Married, Divorce, etc), How Religious (Not Practicing, Moderately Practicing, Fairly Practicing and Very Practicing), Praying (Never Pray, Sometimes Pray, Always Prays and Usually prays), Marriage Plans (As soon as possible, 1-2 years, 3-4 years, 4+ years), whether they consume only halal food, drink alcohol and smoking behaviour. This is because the above characteristics are important for Muslims in the process of selecting a spouse. Sunni and Shi'ite are two factions of Islam which possess differences in certain religious matters such as prayer rituals and belief [33]. As the users of MuzMatch consists of people from different nations, it is crucial to know their sect to avoid any misunderstandings in the future. As for relationship status, it is not permissible for married men or women to engage in infidelity which might lead to adultery. In terms of prayer behaviours, the five daily prayers are obligatory to be performed by every Muslims. It is mentioned in surah An-Nisa, verse 103, Allah says:

"Verily, as-salah (the formal prayer) is

enjoined on the believers at fixed hours."

And Allah says of the people of Hell, in Surah Al-Muddathir, verse 42-43:

"'What has caused you to enter Hell?' They will say:

'We were not of those who used to offer the salah (prayers)."'

As for alcohol and smoking, it is not permissible in Islam as it harms the human body [34].

Apart from the additional features provided by MuzMatch, the users also demonstrated few significant differences on their self-presentation compared to users on Tinder. One of the differences is some of the users included information regarding their family such as their position in the family and number of siblings. This could be due to the purpose of joining the application in order to seek for marriage which is important to provide such information to a potential spouse. In Islam, family members have a very important place for every individual. This is evident in several authentic hadeeths narrated by the Prophet S.A.W. The Prophet S.A.W. said:

"He who believes in Allah and the Last Day, let him maintain good relation with kins" [Al Bukhari and Muslim]".

Another hadith on the importance of having good relationship with family is reported by Anas R.A., Messenger of Allah S.A.W. said:

"Anyone who is pleased that his sustenance is expanded and his age extended should do kindness to his near relatives. [Sunan Abi Dawud]".

Another significant aspect of how Muslims use selfpresentation on Islamic-oriented dating applications is that most of the users talks about marriage or starting a family such as:

"I hope I can find someone to be my life partner", "Seeking a kind, caring, optimisic person... Who will not only be my wife, but also my best friend and companion..." and

"looking for a life partner to complete my deen."

This is related to the nature of the platforms itself whereby Muslim dating applications such as MuzMatch serves as a platform for users to look for potential lifetime spouses which maintains traditional methods of seeking marriage through the feature of adding a chaperone and 
also allows them to preserve their identity as Muslims [35]. This is attributed to the user's motivations to use "application".

In contrast, users on Tinder did not really mention anything related to marriage in the description of their profiles. Instead of mentioning about marriage, some of them only mentioned that they are only looking for romantic partners, friendship, and for fun. Again, the reason is because of the users' intent of using the application. Sumter, et al. [8] reveals that most adult Tinder users used the application for love and casual sex. This is because Tinder has the reputation of allowing users to easily hook up with someone on the internet. Other motivations include ease of communication as it allows people to meet new acquaintances and potential partners easier than in real life situations [36], using Tinder for self-worth validation to get positive feedback on their physical appearance and interests as well as for excitement and trendiness.

\section{Net Presence}

Understanding net presence in Tinder and MusMatch based on the content analysis has yielded some important results. It was revealed that there is no significant difference in terms of their net presence as it relates with one's privacy. The presence of individuals in online dating platforms can only be seen and traced via his/her activities such as chatting, posting pictures, liking or swiping in both Tinder and MuzMatch. According to Ostheimer and Iqbal [37], Tinder, the online dating application has changed and updated their privacy policy for several times as to protect the privacy of its users at all cost. Online dating applications differ from any other social media such as Instagram or WhatsApp that provide the users' net activity to the public. There is no such thing as "online" or "offline" status provided, regardless the type of the platforms.

In both Tinder and MusMatch, there is no sign that indicated users that met face-to-face prior to their virtual presence. Tinder is a platform that secure its users' information including one's net presence and their activities in the application [38]. Dinh et al. [39] revealed that among the daters, one of the ways to acknowledge people's presence is via the "smile" and "liking" of other users' profile. Even though it is an online dating application that can be accessed by everyone, it applies a strict protocols and privacy settings for interaction between genders, especially in Muslim community [40].

\section{Terminal Identities}

The finding of this study towards terminal identities lies with observing "a world behind the screens". This the attribute of terminal identities which dwells on how user perceived behind their screen interactions. That is, the sense and perception created at the interface when interacting with partners in an online dating platforms. Tinder and
MuzMatch, have shown a significant pattern between these two platforms. Tinder does not provide any feature for displaying a clue of the other side of interactions. MuzMatch on the other hand tends to let users to belief that they are connected with a Muslims at the other end of their interaction. That why is widely known as a Muslim-oriented platform which focusses on encouraging halal dating that will lead to marriage as to compare with other mainstream dating platforms. However, in both Tinder and MusMatch, users are allowed to construct their personal identity as to match with their own preferences and interests. It is important to acknowledge that the issue of identity is further complicated as it is hard to disassociate the internet from the sense of anonymity of its users.

Muslim-oriented dating platform is generally perceived as public spaces where individuals who are living in socially conservative non-Western societies, can communicate in order to meet. In MuzMatch dating platform, the way people presented themselves via the profile picture is much respectful and preserved as to compare with Tinder application. People are well aware that majority of the users are Muslims. It was found that the major difference between Muslim and mainstream-oriented dating platforms is, the privacy offered by the Muslim-oriented platform. It is observed that MuzMatch has the option for women to hide their profile picture from the public until they allow them to view it. Here, the self-presentation of oneself via the picture is being guarded and in line with the Islamic perspectives. However, only one out of ten sample of this study that is using this option. According to Sotoudeh et al. [41], Muslimbased online dating users' profile remain unknown and anonymous until the matched users decide to meet offline. Some users believed that profile pictures are not reflecting the reality, therefore it cannot be the initial factor in selecting partner on this platform [42]. Additionally, Musmatch allow its users to use blurry photos as their profile picture. This happens as to prevent the users from making judgement for which is based on one's physical appearance [41]. The analysis of MuzMatch reveals that most of the profile pictures used are in line with their user's personal description about their life. For instance, seven out of ten participants from MuzMatch dating application are using the pictures of their profession.

On the other hand, in Tinder, the users perceived that their profile picture is vital for presenting one's identity. Sumter et al. [8] reveals that, the amount of personal information for conveying one's identity on dating platform is limited, users are most likely to depend on one's appearance through pictures. Profile pictures is use as the key attribute for finding a match. Users decides their match based on profile picture and interests of the potential match. However, according to Turizo [43] it was indicated that even though profile picture is one of the means to trace one's 
online identity, it cannot be denied that the identity can be manufactured as an affected persona. This is done by the users as it helps in facilitating the social interactions. Tinder is generally known as an online avenue where an individual could explore an alternate identity that existed outside of the reality, which had been established as normative behaviour [43]. Tanner and Tabo [44] reveals that in the context of dating environments, individuals are motivated to fully control how they represent themselves and the impression they have created in the eyes of others as to achieve their objective. In western-based dating application, it cannot be denied that the 'profile stage' reflects the period where the users would depict their identities in the excellent manner as for their own benefits.

However, it is undeniable that the users' credibility and authenticity cannot be ascertained in a manipulated profile pictures. It is difficult to confirm the true identity of the person with whom one is communicating with, as people can lie about their profile and identity on both of the dating applications regardless the type of its online environment. It is generally known; individuals were well aware of the veneer truth which is able to be falsified through a person identity construction on this platform [43]. Additionally, in online cyber-dating, regardless of the platform, there is always perceived misinformation and deception relative to personal identity, social status, and other aspects are prevalent. Similarly, Turizo [43] reveals that this cannot be avoided as the media practices related to the social space and setting are closely connected to individual's identity formation.

\section{DISCUSSION}

This study has identified "Terminal identities, Screen names, Net Presence, and Personal Profile" to be the factors influencing online self-presentation associated with Muslimoriented dating and mainstream-oriented dating in platforms.

Terminal identities influence how individual present themselves online. In both MusMatch and Tinder users are allowed to construct their personal identity to match their own preferences and interests. This is the identity created at the interface between the physical body and mobile device or computer. Tinder create a space where it is necessary for individuals to present any form of their identity. This is the identity that the user creates in order to have a presence. Attention to the self is often a goal on dating sites, that is why users are choosing to construct a socially desirable identity that may attract people's attention and publicity. By this, online identities can be real, false, anonymous, or embellished. However, in MusMatch, there is a warning that requires every user to do things based on Islamic approach. There shouldn't be any fake or false information by the members.
The screen name is associated with nickname or pseudonyms which is the beginning of online identity that sets the tone for who controls the content that flows through the platform. MusMatch users screen names drags attentions, because majority of Muslims use Muslims names, therefore, users screen name in MusMatch are mostly Muslims names. Although they can alphanumerical strings which are chosen by the users to form a nick names. They are ubiquitously used for identification and authentication purposes. Tinder users, uses pseudonyms, that is why many of their profile are 'false name'. Dates back during the era of the printing press, 'pseudonymity' became a way for authors or writers to make decisions about how their texts would be known [45]. While in social media, pseudonyms are an important part of identity work, which is the process of creating and contextualizing a self through actions and representation.

In terms of net presence, the frequency with which MusMatch users appear to interact for relationship that leads to marriage is far more than any other form of relationship. Here, the term 'presence' of MusMatch users can be seen by their activities. Thus, it is mostly towards starting a permanent relationship. Net presence represents the extent to which users can know if other users are accessible. It includes knowing where others are, in the virtual world and whether they are available online. To relate, in the virtual world, it happens through status lines like 'available', 'online', 'offline' 'active' or 'hidden.' Given the increasing connectivity of people on the move, this presence bridges virtual communication among the users. Therefore, when relationship is serious, users tend to always watch their presence.

Personal profile of users on both Tinder and MusMatch displays the way users present themselves which may include as much information as they desired about themselves. In MusMatch, personal profile can be either short description or more detailed, depending on oneself, but it has to be the true presentation. Because this provides a piece of information about a person. This is the information that the potential partner will start weighing to determine if a person is trustworthy or not. It is known that this information is generally created by the owner of the account itself. However, in Tinder, although the profile is meant to provide additional information about an individual, the details provided may be based on fantasy or fiction. People are having the chance to create their own personal profile which may consist of age, gender or physical features which are based on their personal preference or filled with tales. Additionally, it is found that Tinder users are creating a virtual identity in which they fashioned an ideal self so that they would impress others. [46].

Self-disclosure is an integral part of presenting oneself online. It refers to a process of communication whereby an 
individual reveals information about themselves to another individual. There is less pressure online as non-verbal cues are absent and people have more time to think before responding to a message. In face-to-face situations, nonverbal cues need to be considered and the other person would expect an immediate respond which creates more tension for the communicator. Online dating plaftforms allows socially shy individuals to easily make friends and also speeds up relationship building process. It is hard for people who are naturally shy to make new friends because it requires them to initiate a conversation and make small talks. With the presence of the internet, the factors that make people socially shy which are direct eye contact, facial expression, tone of voice and such are not present. Therefore, it gives people the ease of making new engagements without the pressure of non-verbal cues [47].

\section{CONCLUSIONS}

The similarities and differences on self-presentation of oneself in two distinct platforms was carried out. The Muslim-oriented and mainstream-oriented online setting has found to influence people in presenting themselves. This study utilized four main themes (Screen names, Personal profiles, Net presence and Terminal identities) for evaluation of the impact of using of online dating platforms. Under the screen names aspect, it was found that both applications allow its users to choose their own username without any restrictions. The platforms showed how screen names can be used as a strategy to present certain impression and image which according to their motives. However, we observed a different pattern in the usage of screen names between the two platforms. It was revealed that only users on MuzMatch used acronyms to identify themselves. Also, apart from using one's real names, it was found that nicknames are commonly used among the sample in both dating applications. Under the personal profile theme, a common trait that can be found on both applications is the description that the users have made in order to describe themselves. There are two distinct description found; which are short and detailed description. Also, it is observed that the users share their own physical attributes, such as height, regardless platform. Users tend to post about their personal interests in order to attract their potential partners on Tinder as well as MuzMatch. In terms of the terminal identities, Tinder users are free to post anything as their profile picture, whereas MuzMatch is so strict to the authenticity of profile pictures. This study has contributed to the understanding of the state of online selfpresentation strategies on dating platforms, and explore how Muslims manage their online presentation on dating approach.

\section{REFERENCES}

[1] B.K. Rajak. "The Future of Social Media in Marketing” Journal of HR, Organizational Behaviour \& Entrepreneurship Development, 3(3), 16, 2020.

[2] E. Mazur, and Y. Li. Identity and self-presentation on social networking web sites: A comparison of online profiles of Chinese and American emerging adults. Psychology of Popular Media Culture, 5(2), 101, 2016.

[3] N. Ellison, R. Heino, and J. Gibbs, "Managing impressions online: Selfpresentation processes in the online dating environment. Journal of computer-mediated communication, 11(2), 415-441, 2006

[4] U. Gündüz. "The effect of social media on identity construction". Mediterranean Journal of Social Sciences, 8(5), 85-92, 2017.

[5] S.C. Herring, and S. Kapidzic. "Teens, gender, and self-presentation in social media". International encyclopedia of social and behavioral sciences, 2, 1-16, 2015.

[6] C. Fullwood, B.M. James, and C.H. Chen-Wilson. "Self-concept clarity and online self-presentation in adolescents. Cyberpsychology, Behavior, and Social Networking, 19(12), 716-720, 2016.

[7] E. Betters, (2017) What is Tinder and How Does it Work? Pocket-lint. [Online] Available: https://www.pocket-

lint.com/apps/news/tinder/139566-what-is-tinder-and-how-does-itwork

[8] S.R Sumter, L. Vandenbosch, and L. Ligtenberg. "Love me Tinder: Untangling emerging adults' motivations for using the dating application Tinder". Telematics and Informatics, 34(1), 67-78, 2017.

[9] M. Graff (2016) The Surprising Facts About Why People Use Tinder. Psychology Today. [Online] Available: https://www.psychologytoday.com/us/blog/lovedigitally/201610/the-surprising-truth-about-why-people-use-tinder

[10] R. Davies (2016) The Young Muslims Finding Love Via an App. Al Jazeera. [Online] Available

https://www.aljazeera.com/indepth/features/2016/10/young muslims-finding-love-app-161018170649390.html

[11] E. Stănculescu, Online self-presentation from the cyberpsychology perspective. In Conference proceedings of» eLearning and Software for Education «(eLSE) (No. 01, pp. 155-160). " Carol I" National Defence University Publishing House. 2011.

[12] L. Jacobson, A. Atkinson, L. Mohamed, and J. Dorr. Singles Looking to Mingle: An Analysis of Self-Presentation in Online Dating. Concordia Journal of Communication Research, 3(1), 2016.

[13] E. Turner, "About Me: A narrative analysis of self-presentation in women's online dating profiles" (Doctoral dissertation), 2012.

[14] N. Ellison, R. Heino, and J. Gibbs, "Managing impressions online: selfpresentation processes in the online dating environment". Journal of Computer-Mediated Communication, 11, 415-441, 2006.

[15] E.M. Morgan, T.C. Richards, and E.M. VanNess, "Comparing narratives of personal and preferred partner characteristics in online dating advertisements". Computers in Human Behavior, 26(5), 883888, 2010.

[16] G. Ranzini and C. Lutz. "Love at first swipe? Explaining Tinder selfpresentation and motives". Mobile Media \& Communication, 5(1), 80-101, 2017.

[17] Shaw, A., \& Sender, K. "Queer technologies: Affordances, affect, ambivalence" Journal Critical Studies in Media Communication, 33(1), 2016 - Queer Technologies, (2016).

[18] N.C. Krämer and S. Winter. "Impression management 2.0: The relationship of self-esteem, extraversion, self-efficacy, and self presentation within social networking sites". Journal of media psychology, 20(3), 106-116, 2008.

[19] J. Ward. "Swiping, matching, chatting: Self-presentation and selfdisclosure on mobile dating apps". Human IT: Journal for Information Technology Studies as a Human Science, 13(2), 81-95, 2016.

[20] W.L. Neuman, "Social Research Methods: Qualitative and quantitative approaches" (7th ed). Boston: Pearson Education, Inc, 2011.

[21] M.D. White and E.E. Marsh. "Content analysis: A flexible methodology". Library trends, 55(1), 22-45, 2006. 
[22] N. Showkat, and H. Parveen, "Non-probability and probability sampling". Media and Communications Study, 1-9, 2017.

[23] K. Black, "Business Statistics: Contemporary Decision Making." (6th ed). Pearson Education Limited, 2010.

[24] A.I. Abubakar, H. Chiroma, S.A. Muaz, and L.B. Ila, A Review of the Advances in Cyber Security Benchmark Datasets for Evaluating DataDriven Based Intrusion Detection Systems. In SCSE (pp. 221-227), 2015.

[25] T. Buchanan, and J.L. Smith. Research on the Internet: Validation of a World-Wide Web mediated personality scale. Behavior Research Methods, Instruments, \& Computers, 31, 565-571, 1999.

[26] M.T. Whitty, "Revealing the 'real'me, searching for the 'actual'you: Presentations of self on an internet dating site". Computers in Human Behavior, 24(4), 1707-1723, 2008.

[27] C. Cobb and T. Kohno. How public is my private life?: Privacy in online dating. In Proceedings of the 26th International Conference on World Wide Web (pp. 1231-1240). 2017

[28] H. Bechar-Israeli, "From to: Nicknames, play, and identity on Internet Relay Chat". Journal of computer-mediated communication, 1(2), JCMC127., 1995.

[29] A. Lakaw (2006). Hiding behind nicknames. A linguistic study of anonymity in IRC chatrooms. [Online] Available Studentische Abschlussarbeit. Växjö University. diva-portal. org. Online verfügbar unter http://www. diva-portal. org/smash/get/diva2, 206935

[30] E.M. Morgan, T.C. Richards and E.M. VanNess, "Comparing narratives of personal and preferred partner characteristics in online dating advertisements. Computers in Human Behavior, 26(5), 883-888, 2010.

[31] A.T. Fiore and J.S. Donath. Online personals: An overview. In CHI'O4 extended abstracts on Human factors in computing systems (pp. 1395-1398, 2004.

[32] E.J. Finkel, P.W. Eastwick, B.R. Karney, H.T. Reis and S. Sprecher. "Online dating: A critical analysis from the perspective of psychological science". Psychological Science in the Public Interest, 13(1), 3-66, 2012.

[33] E. Khalili. Sects in Islam: Sunnis and Shias. International Academic Journal of Humanities, 3(4), 41-47, 2016.

[34] M.J. Byron, J.E. Cohen, J. Gittelsohn, S. Frattaroli, R. Nuryunawati, D.H. Jernigan. "Influence of religious organisations' statements on compliance with a smoke-free law in Bogor, Indonesia: a qualitative study". BMJ open, 5(12), e008111, 2015.

[35] G. Larsson, "Muslims and the New Media: Historical and Contemporary Debates”, UK: Ashgate Publishing Ltd, 2011.
[36] N. Haferkamp, S.C. Eimler, A.M. Papadakis, and J.V. Kruck, Men are from Mars, women are from Venus? Examining gender differences in self-presentation on social networking sites. Cyberpsychology, Behavior, and Social Networking, 15(2), 91-98, 2012.

[37] J. Ostheimer and S. Iqbal. "Privacy in online dating: does it matter? In Proceedings of the 3 rd International Conference on Cryptography, Security and Privacy (pp. 71-75), 2019.

[38] M. Feltz, and M. Chow. "The security of tinder". CsTufts Edu, [Online] Available:

https://media.kasperskycontenthub.com/wpcontent/uploads/sites/4 3/2017/10/20080210/mfeltz.pdf (2015).

[39] R. Dinh, P. Gildersleve, C. Blex, and T. Yasseri. Computationa courtship: understanding the evolution of online dating through large-scale data analysis. arXiv preprint arXiv:1809.10032, 2018.

[40] L. De Rooij. Online matchmaking for British Muslims as the expression of their Islamic identities, [Online] Available https://www.academia.edu/30907761/Online_matchmaking_for_Bri tish_Muslims as the expression_of their Islamic identities $\quad$ L. (2016).

[41] R. Sotoudeh, R. Friedland, and J. Afary. Digital romance: the sources of online love in the Muslim world. Media, Culture \& Society, 39(3), 429-439, 2017.

[42] B. Narin. A Netnography Study About Wapa as a Mobile Dating Application. Journal of Cultural Studies, Faculty of Communication, Hacettepe University, 343-367, 2018.

[43] A.D. Turizo, Constructed Identities and Perpetuated Inequalities in App Dating, [Online] Available, https://repository.upenn.edu/anthro seniortheses/189/., 2018

[44] M. Tanner and P.O. Tabo. "Ladies First: The Influence of Mobile Dating Applications on the Psychological Empowerment of Female Users. Informing Science": The International Journal of an Emerging Transdiscipline, 21, 289-317, 2018.

[45] E. van der Nagel, "Fluids on Pictures on Screens: Pseudonymous Affect on Reddit's TributeMe. Social Media+ Society, 6(1), $2056305120905644,2020$.

[46] M. Sponcil, and P. Gitimu, Use of social media by college students: Relationship to communication and self-concept. Journal of Technology Research, 4, 1, 2013.

[47] M. Chan. Shyness, sociability, and the role of media synchronicity in the use of computer - mediated communication for interpersonal communication. Asian Journal of Social Psychology, 14(1), 84-90, 2011. 